\title{
Development of first-principles interaction model potentials. An application to the study of the bromide hydration
}

\author{
Regla Ayala, José M. Martínez, and Rafael R. Pappalardo \\ Departamento de Química Física, Universidad de Sevilla, 41012-Sevilla, Spain \\ Humberto Saint-Martín and Ivan Ortega-Blake \\ Centro de Ciencias Físicas, Universidad Autónoma de México, Apartado Postal 48-3, Cuernavaca, \\ Morelos 62251, México \\ Enrique Sánchez Marcos ${ }^{\mathrm{a})}$ \\ Departamento de Química Física, Universidad de Sevilla, 41012-Sevilla, Spain
}

(Received 7 June 2002; accepted 17 September 2002)

\begin{abstract}
This work presents the development of first-principles bromide ion-water interaction potentials using the mobile charge density in harmonic oscillators-type model. This model allows for a flexible and polarizable character of the interacting molecules and has already been parametrized for waterwater interactions. The prospected potential energy surfaces of the bromide ion-water system were computed quantum-mechanically at Hartree-Fock and Møller-Plesset second-order perturbation levels. In addition to the ion-solvent molecule pair, structures formed by the anion and two or three water molecules were considered in order to include many body effects. Minimizations of hydrated bromide clusters in gas phase $\left[\mathrm{Br}\left(\mathrm{H}_{2} \mathrm{O}\right)_{n}\right]^{-}(n=1-6,10,15,20)$ and Monte Carlo computations of bromide aqueous solutions were performed to test the new potentials. Both structural and thermodynamic properties have been studied in detail and compared to the available experimental and theoretical values. From these comparisons, it was concluded the importance of including basis set superposition error corrections for the two-body interactions, and the small role of both electron correlation on the three-body terms and the four-body terms. Monte Carlo simulation results have also been used to investigate if the presence of the anion significantly affects the intramolecular geometry of the water molecules and the degree of disruption of the water solvent structure in its vicinity. (C) 2002 American Institute of Physics. [DOI: 10.1063/1.1519843]
\end{abstract}

\section{INTRODUCTION}

Hydration of halides and alkali metals is a subject of interest in solution chemistry and biochemistry. ${ }^{1,2}$ Consequently, there has been a substantial amount of research devoted to understand these systems. A molecular description of these electrolyte aqueous solutions is of fundamental interest, and has stimulated the use of numerical simulations (Monte Carlo and molecular dynamics) ${ }^{3-7}$ as powerful tools for their study. ${ }^{2,8}$ The reliability of these simulations depends critically on the potential model describing the interactions among the particles defining the system. Thus, the development of interaction potentials is currently a topic of increasing importance in both fundamental and applied research. ${ }^{9}$

The development of ion-solvent interaction potentials is an involved task, due to the fact that the most appropriate functional form and the setting of its parameters are not predefined. A widely adopted strategy is to fit the parameters of the ion-water interaction potentials using available experimental information; ${ }^{10}$ however, the good behavior of these empirical potentials is not guaranteed when applied under conditions that considerably differ from those of their parametrization. An alternative strategy for developing potentials is

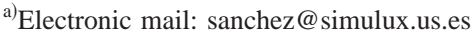

the use of quantum-mechanical information to describe the interaction among particles. Within this framework, the firstprinciples potentials would be of general application, but their development is not exempt from other difficulties that must be overcome, such as the choice of computational level, the region of the surface to be sampled, the computational cost of the sampling, to name but a few.

Precise microscopical descriptions supplied by firstprinciples interaction potentials in combination with adequate statistics for the system under study should ideally lead to describe straightforwardly a wide type of experimental situations, from ionic clusters in gas phase to electrolyte solutions. Apart from the operational difficulties, there are some deeper concerns related to the fact whether the purely quantum-mechanical information extracted from a potential energy surface is able to supply all the ingredients for the macroscopic description. ${ }^{11}$

There has been a great controversy concerning the structure of water molecules around the halide anions. ${ }^{12-47} \mathrm{Sev}$ eral classical simulations ${ }^{14-19,39,48,49}$ have suggested that the halide anions, but fluoride, are attached to the water clusters at the surface instead of being surrounded by water molecules. It has been shown that the results depend critically on the interaction potential considered. Important differences in the structure of the $\left[\mathrm{X}\left(\mathrm{H}_{2} \mathrm{O}\right)_{n}\right]^{-}$are found when they are 
simulated with or without the explicit consideration of the water and ion polarizabilities. $15,17,20,28,29,47-50$

The bromide anion hydration has been widely studied. In a seminal work, Kebarle and Searles ${ }^{51}$ carried out experimental thermodynamic studies of ion solvation in clusters, determining the stepwise addition of solvent molecules to form a solvated ion of increasing size in gas phase. Several works have been focused on this system ${ }^{13,33-35,44,52-59}$ in order to know their structural, spectroscopic and energetic properties. In parallel, a great effort has been made to understand the bromide solvation from a theoretical point of view, studying ionic clusters ${ }^{19,20,22,40-43}$ and bromide aqueous solutions..$^{8,45-47,58,60-66}$ In a recent study about $\mathrm{HBr}$ and $\mathrm{Br}^{-}$ aqueous solvation using $a b$ initio molecular dynamics, Raugei and Klein ${ }^{47}$ postulate an asymmetric solvation shell around the bromide anion indicating a preference for surface states and the need for using a polarizable model to get reliable residence times.

Over the years, several bromide ion-water interaction potentials have been developed. ${ }^{19,58,62,65,67}$ These potentials are able to reproduce experimental properties such as photodetachment energies and provide insight into the controversy about the sort of solvation the bromide anion presents. Nevertheless, none of them is only based on $a b$ initio calculations.

The question here presented is what is the ability of firstprinciples interaction potentials to account simultaneously for the hydration thermodynamics and the microsolvation of the bromide anion? To answer this question $a b$ initio bromide-water interaction potentials are developed in this work. Numerical minimizations of $\left[\mathrm{Br}\left(\mathrm{H}_{2} \mathrm{O}\right)_{n}\right]^{-}$clusters with a small number of water molecules $n=1-6,10,15,20$ and Monte Carlo simulations of the $\mathrm{Br}^{-}$anion in water have been carried out to test the new interaction potentials.

\section{METHODOLOGY}

\section{A. Outline of the MCDHO model}

In the case that solvent-solvent interactions are of the same order as solvent-solute ones, as it is the case of the bromide hydration, ${ }^{50,68}$ the use of a well-balanced model of interactions becomes particularly important. In this work we used a MCDHO-type model (mobile charge densities in harmonic oscillators $)^{69}$ that explicitly includes polarizability and intramolecular flexibility. In the case of the water model, MCDHO reproduces the experimental dipole, ${ }^{70}$ quadrupole ${ }^{71}$ and polarizability ${ }^{72}$ of the molecule. Three positive charges are defined on the atom nuclei positions in the experimental gas-phase geometry of the molecule, with one value for the oxygen atom, $Z_{\mathrm{O}}$, and another for each hydrogen atom, $Z_{\mathrm{H}}$. A negative mobile charge, $q_{\mathrm{O}}=-Z_{\mathrm{O}}-2 Z_{\mathrm{H}}$, attached to the oxygen atom by a harmonic oscillator is used to model the polarizability (see Fig. 1). In order to avoid the so-called polarization catastrophe $e^{73}$ it is necessary to screen the Coulombic interaction by considering the mobile charge $q_{\mathrm{O}}$ as a spherical charge density $\rho_{\mathrm{O}}$ with radial exponential decay [see Eq. (1) of Ref. 69]. The intramolecular flexibility of water molecules is included by means of the electrostatic interaction among the charges, except the interaction be-

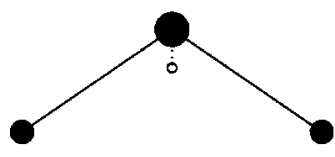

water molecule

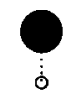

bromide ion
FIG. 1. Schematic representation of MCDHO model. Positive charges $Z_{O}$, $\mathrm{Z}_{\mathrm{H}}$ and $\mathrm{Z}_{\mathrm{Br}^{-}}$are placed at the nuclei positions (bold sphere), and the mobile charges $q_{\mathrm{O}}$ and $q_{\mathrm{Br}^{-}}$are attached to the oxygen atom and the bromide atom by a harmonic oscillator potential (white sphere).

tween the mobile charge and the charged nucleus to which it is attached, a Morse potential for the $\mathrm{O}-\mathrm{H}$ bonds, and a fourth degree polynomial for the $\mathrm{HOH}$ angle.

The inclusion of intramolecular interactions results in a nonzero energy for each molecule $n$; the intramolecular energy produced by the model for an isolated molecule, $U_{n}^{\circ}$, is used as a reference to account for the energetic cost, $\Delta U_{n}$, of the polarization and the deformation induced when the molecule interacts with others, changing its energy to $U_{n}$, whence $\Delta U_{n}=U_{n}-U_{n}^{\circ}$. In the case of the bromide, a monoatomic ion is described by a positive charge $Z_{\mathrm{Br}^{-}}$on its nucleus and a mobile negative charge density $\rho_{\mathrm{Br}^{-}}$with total charge $q_{\mathrm{Br}^{-}}=-Z_{\mathrm{Br}^{-}}-1$, attached to it (see Fig. 1). The intra-atomic energy is defined by $U_{\mathrm{Br}^{-}}=\frac{1}{2} k_{\mathrm{Br}^{-}} r^{2}$, where $r$ is the distance between the core and its associated mobile charge density. In the absence of an external field, the equilibrium position of the oscillator is located on the nucleus, thence $U_{\mathrm{Br}^{-}}^{\circ}=0$.

The water-water interactions were described by the MCDHO potential already published. ${ }^{69}$ The bromide ionwater interaction potential has been developed in this work by considering the following intermolecular terms:

1 A classical 12-6-1 potential between the negative mobile charges $q_{\mathrm{O}}$ and $q_{\mathrm{Br}^{-}}, r$ being the distance between them, and $A$ and $B$ the Lennard-Jones parameters,

$$
U\left(q_{\mathrm{O}}, q_{\mathrm{Br}^{-}}\right)=\left(\frac{A_{\mathrm{Br}-\mathrm{O}}}{r}\right)^{12}-\left(\frac{B_{\mathrm{Br}-\mathrm{O}}}{r}\right)^{6}+\frac{q_{\mathrm{O}} q_{\mathrm{Br}^{-}}}{r} .
$$

2 An electrostatic interaction between $\rho_{\mathrm{O}}$ and $Z_{\mathrm{Br}^{-}}, r^{\prime}$ being the distance between the center of $\rho_{\mathrm{O}}$ and the nucleus of bromide,

$U\left(q_{\mathrm{O}}, Z_{\mathrm{Br}^{-}}\right)=\frac{q_{\mathrm{O}} Z_{\mathrm{Br}^{-}}}{r^{\prime}}\left[1-\left(\frac{r^{\prime}}{\lambda_{\mathrm{O}}^{\prime}}+1\right) \exp \left(-2 \frac{r^{\prime}}{\lambda_{\mathrm{O}}^{\prime}}\right)\right]$,

where $\lambda_{\mathrm{O}}^{\prime}$ corresponds to the intermolecular screening, that is different from the intramolecular one, $\lambda_{\mathrm{O}}$, both of them described in Ref. 69.

3 An electrostatic interaction between $\rho_{\mathrm{Br}^{-}}$and each of the charges on the nuclei of the water molecule $Z_{i}(i \equiv \mathrm{O}, \mathrm{H})$ :

$$
U\left(Z_{i}, q_{\mathrm{Br}^{-}}\right)=\frac{Z_{i} q_{\mathrm{Br}^{-}}}{r_{i}}\left[1-\left(\frac{r_{i}}{\lambda_{\mathrm{Br}^{-}}^{\prime}}+1\right) \exp \left(-2 \frac{r_{i}}{\lambda_{\mathrm{Br}^{-}}^{\prime}}\right)\right] \text {, }
$$

where $r_{i}$ is the distance from the center of $\rho_{\mathrm{Br}^{-}}$to $Z_{i}$, and the $\lambda_{\mathrm{Br}^{-}}^{\prime}$ notation has been maintained for intermolecular interactions.

4 A potential between the bromide nucleus and each of the nuclei of the water molecule, that includes the electrostatic 
repulsion of their respective point-charges, and has two additional terms:

$U\left(Z_{i}, Z_{\mathrm{Br}^{-}}\right)=\frac{C_{\mathrm{Br}-i}}{R_{i}^{s_{\mathrm{Br}-i}}}-\frac{D_{\mathrm{Br}-i}}{R_{i}^{p_{\mathrm{Br}-i}}}+\frac{Z_{i} Z_{\mathrm{Br}^{-}}}{R_{i}}$,

where $\mathrm{C}_{\mathrm{Br}-\mathrm{O}}, \mathrm{C}_{\mathrm{Br}-\mathrm{H}}, \mathrm{D}_{\mathrm{Br}-\mathrm{O}}, \mathrm{D}_{\mathrm{Br}-\mathrm{H}}, \mathrm{s}_{\mathrm{Br}-\mathrm{O}}, \mathrm{s}_{\mathrm{Br}-\mathrm{H}}, \mathrm{p}_{\mathrm{Br}-\mathrm{O}}$, $\mathrm{p}_{\mathrm{Br}-\mathrm{H}}$, are adjustable parameters, and $R_{i}$ is the distance from the bromide nucleus to the $i$-th nucleus of the water molecule. The use of the exponents $s_{i}$ and $p_{i}$ as free parameters was based on previous studies ${ }^{74,75}$ that showed the advantage of using exponents different from 12 and 6 to reproduce $a b$ initio data. In this work the exponents $s_{i}$ and $p_{i}$ were not constrained to be integers.

\section{B. Quantum-mechanical calculations}

The exploration of potential energy surfaces for the bromide anion-water system was carried out at the MP2 level using the GAUSSIAN 98 program. ${ }^{76}$ The bromide anion was described by a DZ basis set ${ }^{77}$ augmented by polarization and sp diffuse functions. $^{78}$ For water molecules the aug-cc-pVDZ ${ }^{79,80}$ basis set was used. It is worth pointing out that this basis set is able to reproduce the gas phase dipole moment of the water molecule $(1.99 \mathrm{D})$ in good agreement with the experimental results $(1.85 \mathrm{D})$. This is interesting because ion-dipole interactions represent a significant contribution to the long-range interactions of the bromide ion-water. ${ }^{81}$

Potential energy surfaces corresponding to $\mathrm{Br}\left(\mathrm{H}_{2} \mathrm{O}\right)^{-}$, $\mathrm{Br}\left(\mathrm{H}_{2} \mathrm{O}\right)_{2}^{-}$and $\mathrm{Br}\left(\mathrm{H}_{2} \mathrm{O}\right)_{3}^{-}$were scanned to account for not only pairwise interaction energies, but also for nonadditive contributions.

For dimers, $\mathrm{Br}\left(\mathrm{H}_{2} \mathrm{O}\right)^{-}$, several scans were performed varying the $\mathrm{Br}-\mathrm{O}$ distance, $\mathrm{BrHO}$ angle $(\gamma)$ and $\mathrm{BrHOH}$ dihedral angle $(\phi)$ (Scheme 1). The pairwise interactions were computed by the usual expression:

$$
E^{(2)}\left(X_{1}, X_{2}\right)=E\left(X_{1}, X_{2}\right)-E^{(1)}\left(X_{1}\right)-E^{(1)}\left(X_{2}\right),
$$

where the reference geometry of water molecule was the experimental one.

The nonadditive contributions to the total interaction energy were calculated as follows:

$$
\begin{aligned}
E^{(3)} & \left(X_{1}, X_{2}, X_{3}\right) \\
& =E\left(X_{1}, X_{2}, X_{3}\right)-\sum_{i=1}^{3} E^{(1)}\left(X_{i}\right)-\sum_{i=1, j>i}^{3} E^{(2)}\left(X_{i}, X_{j}\right)
\end{aligned}
$$

for the three-body contributions, and

$$
\begin{aligned}
E^{(4)}\left(X_{1}, X_{2}, X_{3}, X_{4}\right) \\
=E\left(X_{1}, X_{2}, X_{3}, X_{4}\right)-\sum_{i=1}^{4} E^{(1)}\left(X_{i}\right)-\sum_{i=1, j>i}^{4} E^{(2)}\left(X_{i}, X_{j}\right) \\
\quad-\sum_{i=1, j>i, k>i, j}^{4} E^{(3)}\left(X_{i}, X_{j}, X_{k}\right)
\end{aligned}
$$

for the four-body contributions.

The geometries of trimers and tetramers were taken from a previous quantum-mechanical work ${ }^{68}$ concerning the microsolvation of the bromide anion. They present water molecular geometries distorted by the presence of the ion. In this case, $E^{(2)}\left(X_{1}, X_{2}\right)$ is computed by Eq. (5) using as reference geometry of water molecules, the distorted ones in the trimers and tetramers. This procedure is particular suitable to warranty a good convergence of the many-body interactions development, as the adopted partition includes the geometrical distortion of water in the $E^{(i)}\left(X_{i}\right)$ terms.

\section{Fitting procedure}

In order to ascertain the level of theory that must be used to get a reliable interaction potential, the following points were considered:

(i) The influence of the basis set superposition error (BSSE) on pairwise interaction energies. The BSSE was calculated by the counterpoise method ${ }^{82}$ Although its inclusion improves the interaction energies estimation, there is a controversy about to what extent BSSE corrections improve the reliability of the results. ${ }^{30,83-86}$

(ii) The influence of BSSE correction, electron correlation and many-body terms on nonadditivity contributions. The BSSE influence on nonadditivity is not clear, especially in the case of the anions. ${ }^{81}$ In order to decide the nonadditivity treatment, 19 different trimers were studied quantummechanically. They were selected so that the trimer surface sampled was representative, that is, energetic and structural selection criteria were considered to choose the trimers studied. The comparison of the SCF, SCF BSSE corrected, MP2 and MP2 BSSE corrected results showed that both electron correlation and BSSE correction have a small effect on the three-body contributions (differences are in the hundredth of kilocalorie). The small effect of including electron correlation is not surprising because it is known that this contribution is strongly additive. ${ }^{87}$ Nevertheless, the influence of these small variations on the results of the Monte Carlo simulations and numerical minimizations is not clear, so we will proceed to check it. In a previous work ${ }^{68}$ dealing with the microsolvation of the bromide ion in different solvents, a
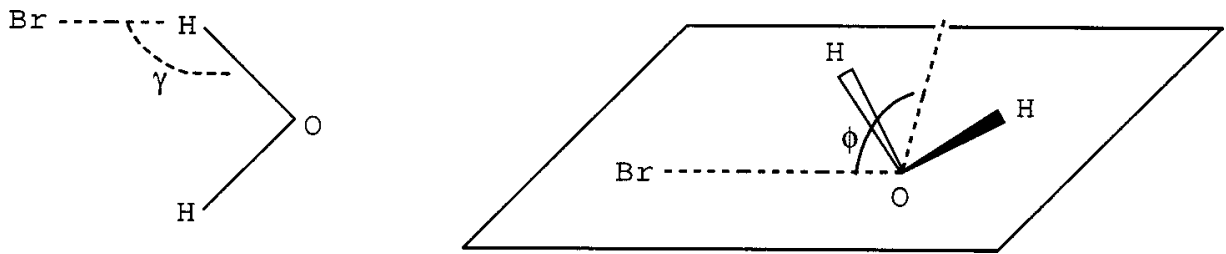

Scheme of the $\angle \mathrm{BrHO}$ angle, $(\gamma)$ and the tilt angle, $(\phi)$. 
TABLE I. Summary of the characteristics for the interaction potentials developed.

\begin{tabular}{llll}
\hline \hline \multicolumn{1}{c}{ Interaction Type } & POT_1 & \multicolumn{1}{c}{ POT_2 } & \multicolumn{1}{c}{ POT_3 } \\
\hline Two-body nonadditivity & MP2 $^{\mathrm{a}}$ & MP2 BSSE $^{\mathrm{b}}$ & MP2 BSSE \\
Three-body nonadditivity & MP2 & MP2 & SCF $^{\mathrm{a}}$ \\
Four-body nonadditivity & NO & NO & SCF \\
\hline
\end{tabular}

$\overline{{ }^{a} \mathrm{MP} 2 \text { or SCF denotes the calculation level employed to obtain the quantum }}$ mechanical energy of each interaction type.

${ }^{\mathrm{b}} \mathrm{BSSE}$ means that the basis-set superposition error was corrected by the counterpoise method.

partial cancellation of many-body contributions when computing the interaction energy was found. The solute-solvent and the solvent-solvent contributions almost cancel each other, though they are large in magnitude. These results agree with those recently obtained by Kim et al. ${ }^{42}$ An examination of different contributions shows that three-body terms are the main responsible for the nonpairwise additive character of the interactions. What's more, it has been demonstrated that the inclusion of the three-body effects is important for a more accurate description of the solvation structure. ${ }^{88,89}$ Four-body contributions are by far less important and they imply a considerably larger computational effort.

Bearing in mind the previous considerations, three different bromide ion-water interaction potentials were devel- oped quantum-mechanically. They have been defined in order to learn which of the mentioned effects are important for a proper description of the ion hydration, as well as to find a compromise between the accuracy of the description and the computational cost of building the potential:

POT_1 was obtained by fitting to the functional form of Eqs. (1)-(4), the MP2 energies corresponding to the pairwise interactions and three-body contributions, none of them BSSE corrected.

POT_2 was fitted to the MP2 pairwise interactions BSSE corrected, whereas three-body contributions remained uncorrected. In this case, the counterpoise ${ }^{82}$ correction for the three-body terms, which is much more time consuming than that of the two-body terms, is avoided.

POT_3 was fitted to the MP2 pairwise interactions BSSE corrected, whereas three- and four-body nonadditivities were considered only at the SCF level and were not BSSE corrected. In this case, we have taken into account the behavior of the result of a previous study on 19 trimers, mentioned in point (ii), that suggests how nonadditivity can be accurately obtained at the SCF level.

Table I summarizes the characteristics of the different potentials.

The total number of geometries evaluated in the fitting procedure was 538 dimers, 125 trimers and 76 tetramers. They were adjusted taking into account a nonlinear fitting. Figure 2 plots a goodness of the fitting procedures, by show-
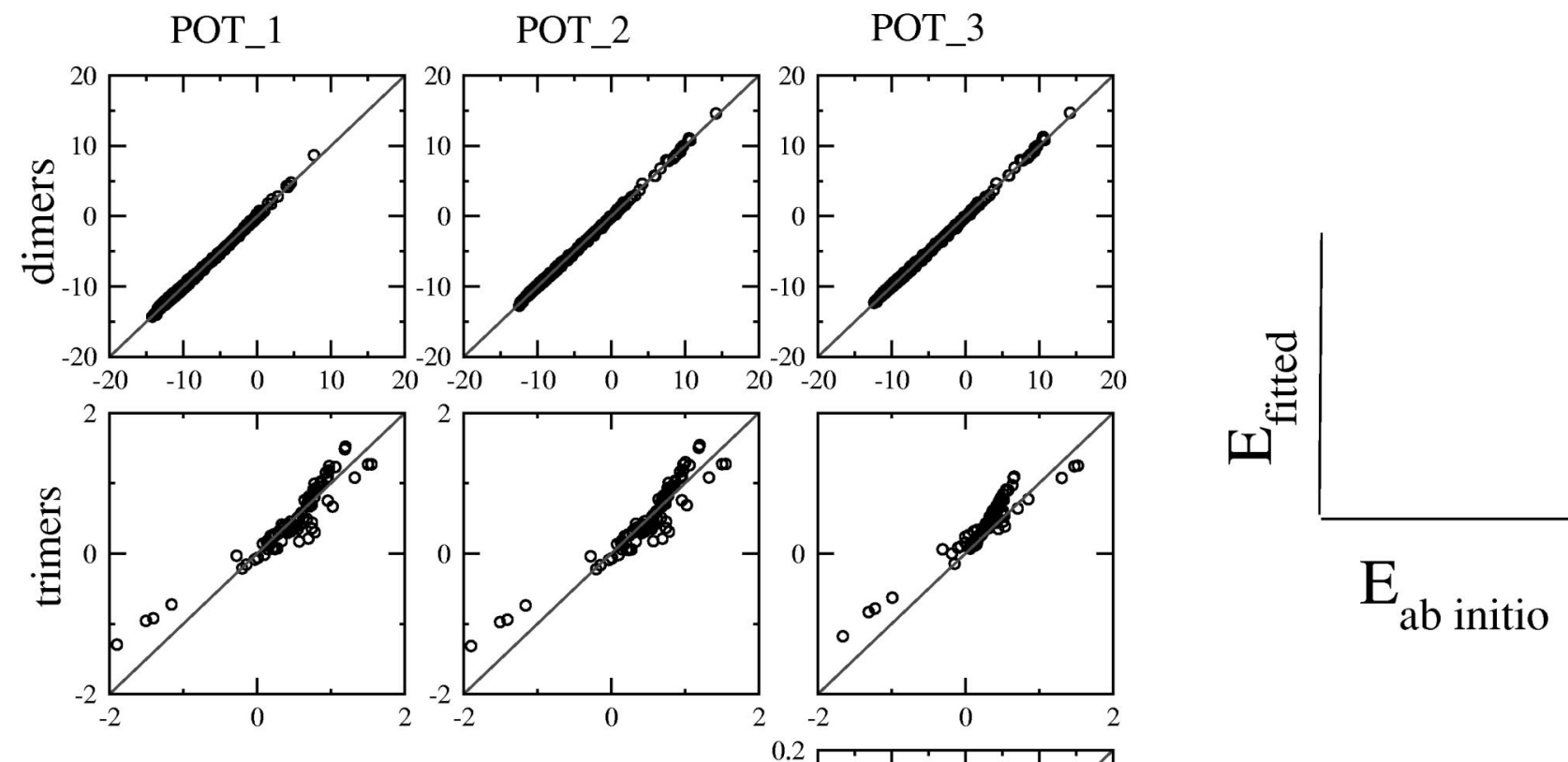

进

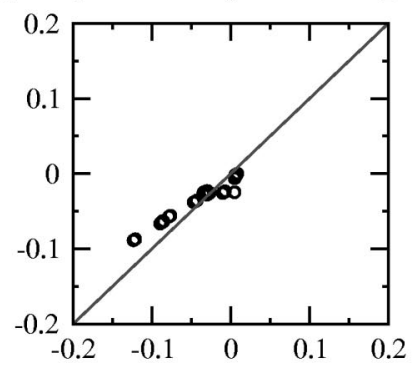

FIG. 2. Plots of fitted vs $a b$ initio energy points of the pairwise and three- and four-body contributions for POT_1, POT_2 and POT_3. The straight lines with slope 1 are put for reference only, and do correspond to minimum squares graphs of the points. All the values are in $\mathrm{kcal} / \mathrm{mol}$. 
ing the high correlation between the $a b$ initio energies and those predicted by the potentials. The parameters for each of the above potentials as well as the standard deviations of the corresponding fitting are presented in Table II.

\section{Minimizations and Monte Carlo numerical simulations}

Numerical minimizations and Monte Carlo simulations were carried out using the MCHANG program developed by the Cuernavaca group. ${ }^{90}$

For $\left[\mathrm{X}\left(\mathrm{H}_{2} \mathrm{O}\right)_{n}\right]^{-}$clusters $(\mathrm{X} \equiv \mathrm{F}, \mathrm{Cl}, \mathrm{Br}, \mathrm{I})$ different structures can be found, all of them being minima and quite close in energy. $31,32,41-43,68$ Moreover, the number of possible structures increases with the size of the cluster, ${ }^{30,68,91}$ due to the significant role played by the water-water interactions. In order to guarantee an adequate prospecting, numerical energy minimizations using different strategies were carried out. On one hand, random geometries generated by heating the system were considered. On the other hand, starting points close to the quantum-mechanical minima ${ }^{68}$ were also considered.
TABLE II. Parameters of the three model potentials described in the text (Sec. II C), in a.u. The three models have the same functional form [Eqs. (1)-(4)], but were fitted to different sets of data (Table I). The standard deviations $\sigma$ are in $\mathrm{kcal} / \mathrm{mol}$ and show the degree of confidence of the fitting.

\begin{tabular}{lccc}
\hline \hline Parameters & POT_1 & POT_2 & POT_3 \\
\hline$Z_{\mathrm{Br}}$ & 9.5337469 & 7.2420000 & 6.8395841 \\
$q_{\mathrm{Br}}$ & -10.5337469 & -8.2420000 & -7.8395841 \\
$k$ & 3.0573096 & 1.7365385 & 2.2932305 \\
$A_{\mathrm{Br}-\mathrm{O}}$ & 3.284519426 & 3.163838821 & 3.232721837 \\
$B_{\mathrm{Br}-\mathrm{O}}$ & 1.205742066 & 1.224004886 & 1.265076834 \\
$\lambda_{\mathrm{Br}-}^{\prime}$ & 0.67142865 & 0.69748352 & 0.70474811 \\
$C_{\mathrm{Br}-\mathrm{O}}$ & 0 & 0 & 0 \\
$s_{\mathrm{Br}-\mathrm{O}}$ & 0 & 0 & 0 \\
$D_{\mathrm{Br}-\mathrm{O}}$ & 8.9581148 & 5.9276008 & 6.3714829 \\
$p_{\mathrm{Br}-\mathrm{O}}$ & 4.1899265 & 4.3000000 & 4.3000000 \\
$C_{\mathrm{Br}-\mathrm{H}}$ & 4234.0284 & 4202.6245 & 4219.5900 \\
$s_{\mathrm{Br}-\mathrm{H}}$ & 12.007719 & 12.500000 & 12.000000 \\
$D_{\mathrm{Br}-\mathrm{H}}$ & 21.164999 & 8.7851380 & 13.133671 \\
$p_{\mathrm{Br}-\mathrm{H}}$ & 6.0152096 & 6.0000000 & 6.0000000 \\
& \multicolumn{2}{c}{ Standard deviations $\sigma$} & \\
& 0.1810 & 0.1240 & 0.1442 \\
$\sigma$ 2-body & 0.1851 & 0.1859 & 0.1871 \\
$\sigma$ 3-body & - & - & 0.0170 \\
$\sigma$ 4-body & - & \\
\hline \hline
\end{tabular}

TABLE III. Interaction energies in $\mathrm{kcal} / \mathrm{mol}$ of $\left[\mathrm{Br}\left(\mathrm{H}_{2} \mathrm{O}\right)_{n}\right]^{-}$clusters. Comparison between results calculated using the interaction potentials developed and ab initio methods. The (n.m) notation means that there are "n" water molecules at one side of the bromide ion and " $\mathrm{m}$ " at the opposite side. Values in parentheses correspond to interaction energies when the clusters geometry is relaxed within the force field of the interaction potentials developed. Some of the minima obtained are collected in Fig. 3 (see clusters (a.x), (c.x) and (g.x) in Fig. 3)

\begin{tabular}{ccccc}
\hline \hline$n$ & POT_1 & POT_2 & POT_3 & ab initio \\
\hline 1 & -13.90 & -12.03 & -11.96 & $-13.88^{\mathrm{a}}-12.46^{\mathrm{b}}-12.7 \pm 0.9^{\mathrm{c}}$ \\
& $(-14.02)$ & $(-12.03)$ & $(-11.96)$ & \\
$(2.0)$ & -28.64 & -25.21 & -24.87 & $-28.54^{\mathrm{a}}-25.26^{\mathrm{b}}-25.8 \pm 3.1^{\mathrm{c}}$ \\
& $(-28.90)$ & $(-25.22)$ & $(-24.89)$ & \\
$(2.1)$ & -39.10 & -34.18 & -34.26 & $-39.42^{\mathrm{a}}-34.71^{\mathrm{b}}$ \\
& $(-42.69)$ & $(-37.48)$ & $(-36.96)$ & \\
$(3.0)$ & -44.77 & -39.38 & -38.78 & $-44.63^{\mathrm{a}}-38.60^{\mathrm{b}}-39.7 \pm 3.6^{\mathrm{c}}$ \\
& $(-45.15)$ & $(-39.89)$ & $(-39.35)$ & \\
$(2.2)$ & -50.80 & -44.68 & -44.98 & $-51.52^{\mathrm{a}}-45.15^{\mathrm{b}}-47.0 \pm 3.5^{\mathrm{c}}$ \\
& $(-58.70)$ & $(-52.42)$ & $(-52.01)$ & \\
$(3.1)$ & -52.50 & -46.30 & -46.66 & $-53.45^{\mathrm{a}}-46.63^{\mathrm{b}}$ \\
& $(-56.38)$ & $(-49.83)$ & $(-52.01)$ & \\
$(4.0)$ & -56.98 & -51.24 & -50.85 & $-57.73^{\mathrm{a}}-50.09^{\mathrm{b}}-52.6 \pm 5.0^{\mathrm{c}}$ \\
& $(-58.70 \mathrm{a} .1)$ & $(-52.42 \mathrm{a} .2)$ & $(-52.01 \mathrm{a} .3)$ & \\
$(3.2)$ & -63.33 & -56.02 & -56.78 & $-64.76^{\mathrm{a}}-56.24^{\mathrm{b}}-56.88^{\mathrm{d}}$ \\
$(4.1)$ & $(-67.77)$ & $(-60.12)$ & $(-59.43)$ & \\
& -65.19 & -58.08 & -58.68 & $-66.71^{\mathrm{a}}-58.16^{\mathrm{b}}-58.95^{\mathrm{d}}$ \\
$(5.0)$ & $(-69.07)$ & $(-62.83)$ & $(-64.39)$ & $-69.91^{\mathrm{a}}-61.03^{\mathrm{b}}-61.34^{\mathrm{d}}$ \\
& -69.17 & -62.98 & -62.45 & \\
$(3.3)$ & $(-72.19 \mathrm{c} .1)$ & $(-65.07 \mathrm{c} .2)$ & $(-64.41 \mathrm{c} .3)$ & \\
& -76.02 & -67.50 & -68.45 & $-77.88^{\mathrm{a}}-67.30^{\mathrm{b}}-67.74^{\mathrm{d}}$ \\
& $(-78.19 \mathrm{~g} .1)$ & $(-69.85 \mathrm{~g} .2)$ & $(-70.44 \mathrm{~g} .3)$ & \\
\hline
\end{tabular}

${ }^{a}$ Single Point Interaction Energies at MP2 on geometries optimized at B3LYP (Ref. 68) level without BSSE correction.

${ }^{\mathrm{b}}$ Single Point Interaction Energies at MP2 on geometries optimized at B3LYP (Ref. 68) level including BSSE correction.

${ }^{\mathrm{c}} \mathrm{MP} 2$ interaction energies from Ref. 42 following the scheme: $\Delta E_{e}=\left(\Delta E_{e}^{N}+\Delta E_{e}^{B}\right) / 2 \pm \mathrm{BSSE} / 2$, where $\Delta E_{e}^{N}$ and $\Delta E_{e}^{B}$ are interaction energies without and with BSSE correction.

${ }^{\mathrm{d}}$ BLYP interaction energies from Ref. 43 following the scheme: $\Delta E_{e}+50 \%$ BSSE correction. 


\section{POT_1}

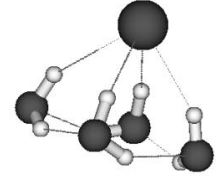

a.1 $\mathrm{n}=4 \mathrm{E}_{\text {int }}=-58.7$

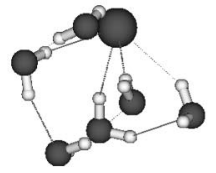

e.1 $\mathrm{n}=6 \mathrm{E}_{i n t}=-85.4$

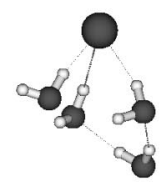

b.1 $\mathrm{n}=4 \mathrm{E}_{\text {int }}=-56.5$

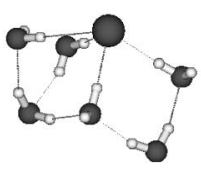

f. $1 \mathrm{n}=6 \mathrm{E}_{\text {int }}=-80.0$

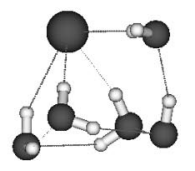

c. $1 \mathrm{n}=5 \mathrm{E}_{\text {int }}=-72.2$

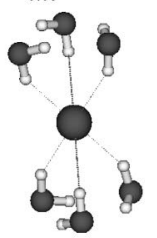

g.1 $\mathrm{n}=6 \mathrm{E}_{\text {int }}=-78.2$

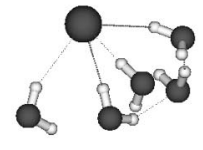

d. $1 \mathrm{n}=5 \mathrm{E}_{\text {int }}=-69.1$

POT_2
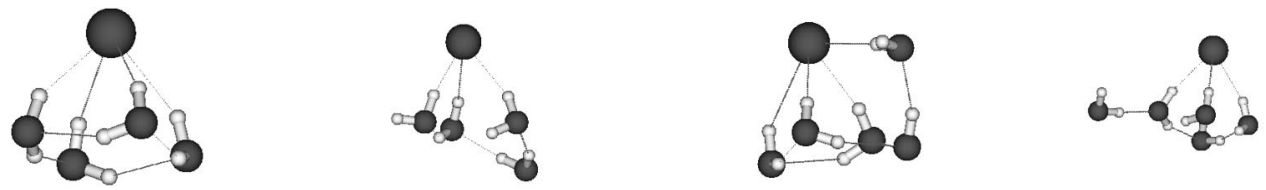

a.2 $\mathrm{n}=4 \mathrm{E}_{\text {int }}=-52.4$

b. $2 \mathrm{n}=4 \mathrm{E}_{\text {int }}=-50.6$
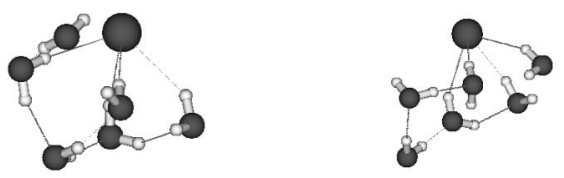

$\mathrm{e} .2 \mathrm{n}=6 \mathrm{E}_{\text {int }}=-77.3$

$\mathrm{f} .2 \mathrm{n}=6 \mathrm{E}_{\text {int }}=-73.9$

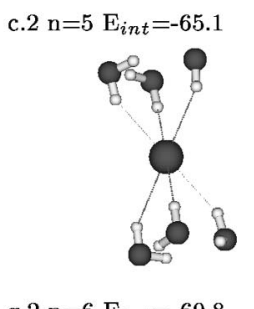

g. $2 \mathrm{n}=6 \mathrm{E}_{\text {int }}=-69.8$

POT_3
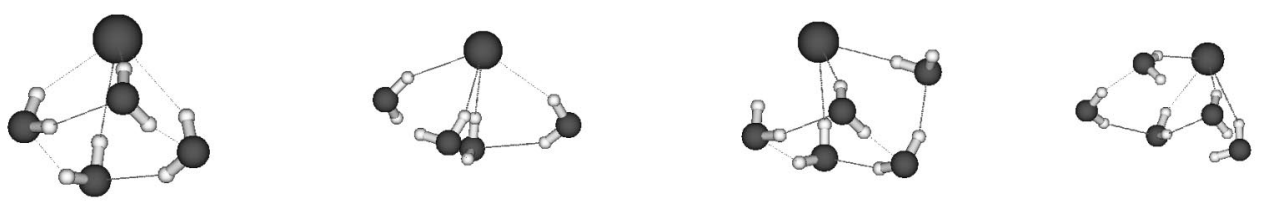

a.3 $\mathrm{n}=4 \mathrm{E}_{\text {int }}=-52.0$

b. $3 \mathrm{n}=4 \mathrm{E}_{\text {int }}=-49.2$

$\mathrm{d} .3 \mathrm{n}=5 \mathrm{E}_{i n t}=-61.6$

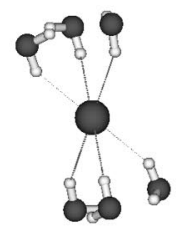

e. $3 \mathrm{n}=6 \mathrm{E}_{i n t}=-76.6$

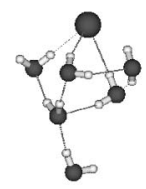

$\mathrm{f} .3 \mathrm{n}=6 \mathrm{E}_{i n t}=-74.6$

g. $3 \mathrm{n}=6 \mathrm{E}_{\text {int }}=-70.4$

FIG. 3. Optimized minimum energy geometries of $\left[\mathrm{Br}\left(\mathrm{H}_{2} \mathrm{O}\right)_{n}\right]^{-}$clusters $(n=4-6)$ obtained with POT_1 (first row), POT_2 (second row) and POT_3 (third row). The interaction energies are in $\mathrm{kcal} / \mathrm{mol}$.

Monte Carlo simulations of 211 water molecules and 1 bromide anion in the NVT ensemble, at a temperature of $T$ $=298.15 \mathrm{~K}$ were also performed in order to compare the different potentials generated. Periodic boundary conditions and a spherical cutoff radius of $R_{\text {cut }}=\mathrm{L} / 2 \AA$ were used for a cubic box of side length $L=18.76 \AA$. Long-range interactions have been treated by means of the Ewald sum technique ${ }^{3}$ including the charged system term. ${ }^{92,93}$ Although 


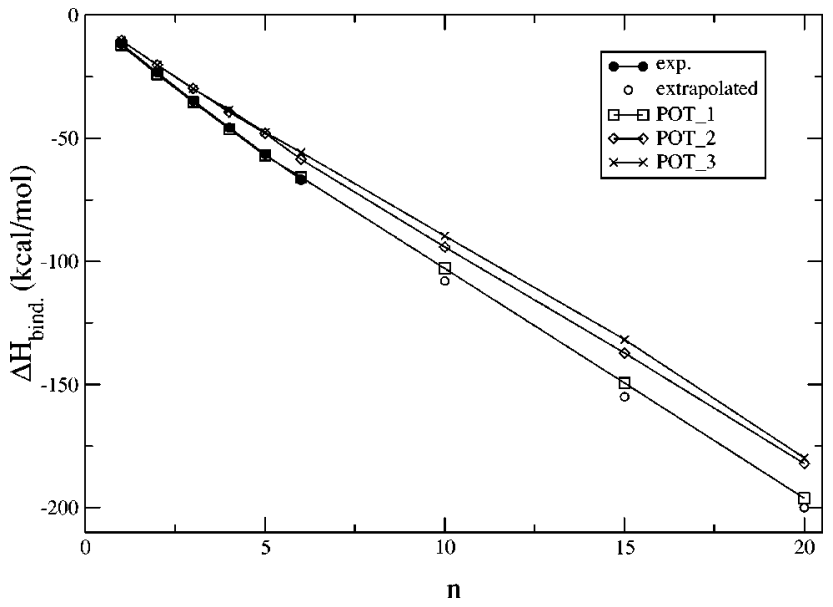

FIG. 4. Binding enthalpies for the formation reaction of the ionic cluster at $300 \mathrm{~K}$. Values are in $\mathrm{kcal} / \mathrm{mol}$. Experimental uncertainties are $0.3-0.4 \mathrm{kcal} /$ mol per water molecule.

this treatment is costly, its importance to obtain reliable thermodynamic results has been proven in the case of ionic solutions. ${ }^{92,94-98}$ A Metropolis ${ }^{99}$ algorithm was implemented with $\sim 40 \%$ of acceptance of Monte Carlo moves. The starting configuration was generated from a liquid water simulation where five molecules were replaced by a bromide anion in order to maintain the water density $\rho=0.997 \mathrm{~g} / \mathrm{cm}^{3}$. Different starting points were considered for a set of runs. The convergence achieved in all cases revealed that the initial configuration has no effect on the results. Large equilibration periods were carried out ( $300 \mathrm{M}$ configurations) because the inclusion of the intramolecular flexibility and polarizability results in a large number of degrees of freedom.

The capability of the Ewald summation treatment to provide size consistent hydration enthalpy for ionic systems was tested by performing additional Monte Carlo simulations under similar conditions as previously described of 1 bromide anion with 116 and 507 water molecules. The results are presented in the following section.

\section{RESULTS AND DISCUSSION}

The potentials generated are tested in this section. Results derived from minimizations of clusters and Monte Carlo simulations which employed the new potentials are compared to both experimental results and previous theoretical computations. From the results of the numerical simulations, the hydration structure around the anion is determined, and the hydrogen bonding in the liquid and the average value of the dipole moment of the water molecules are analyzed.

\section{A. Minimizations of $\left[\mathrm{Br}\left(\mathrm{H}_{2} \mathrm{O}\right)_{n}\right]^{-}$}

The interest in the comparison between the interaction potentials and ab initio calculations lies on proving the ability of the potentials to reproduce clusters that present a larger size than those included in the potential energy surfaces sampled during the potential development. Using optimized structures obtained in Ref. 68, quantum-mechanical single point calculations for $\left[\mathrm{Br}\left(\mathrm{H}_{2} \mathrm{O}\right)_{n}\right]^{-}$clusters were performed. They were carried out at the same level of that used for the prospecting of the bromide-water system. The interaction energies were BSSE corrected by the counterpoise procedure. In parallel, the interaction energy for these structures was computed using one of the bromide-water potentials (POT_i, $i \equiv 1,2$ and 3 ) and the water-water MCDHO potential.

Table III shows the comparison between the interaction energy computed from $a b$ initio calculations and from the intermolecular potentials developed. There is resemblance in the comparison between the interaction energies BSSE corrected and POT_2 and POT_3. Similarly, POT_1 results are close to the noncorrected BSSE energies. Values for the same kind of clusters, optimized at other ab initio level by Kim and colleagues, ${ }^{42,43}$ have also been included in Table III. The

TABLE IV. Summary of the structural and energetic results obtained with different potentials, AIMD simulations and experimental results. Average polarizability, $\alpha_{\mathrm{Br}^{-}}$, in $\AA^{3}$; average dipole moment, $\mu_{\mathrm{Br}^{-}}$, in Debyes; distances in $\AA$; angles in degrees and enthalpies in $\mathrm{kcal} / \mathrm{mol}$. The values in parentheses are the $\Delta H_{\text {hyd }}$ that were obtained from simulations where the Ewald sum was not applied.

\begin{tabular}{|c|c|c|c|c|c|}
\hline Parameter & POT_1 & POT_2 & POT_3 & $\begin{array}{l}a b \text { initio } \\
\text { M. D. }{ }^{\text {a }}\end{array}$ & Exp. $^{\text {b }}$ \\
\hline$\alpha_{\mathrm{Br}^{-}}$ & 6.74 & 5.80 & 5.52 & & 4.85 \\
\hline$\mu_{\mathrm{Br}^{-}}$ & & $0.8-1.0$ & & 1.0 & \\
\hline $\mathrm{BrHO}$ angle $(\gamma)$ & & 177.5 & & & \\
\hline Tilt angle $(\theta)$ & & 47.0 & & 52.0 & \\
\hline max. of $g_{\mathrm{Br}-\mathrm{O}}(r)$ first peak & 3.35 & 3.42 & 3.43 & 3.37 & $3.30-3.43$ \\
\hline Oxygens in the first shell & 10.0 & 7.2 & 7.0 & 7.5 & $6-8$ \\
\hline max. of $g_{\mathrm{Br}-\mathrm{H}}(r)$ first peak & 2.37 & 2.44 & 2.50 & 2.32 & \\
\hline Hydrogens in the first shell & 5.86 & 5.76 & 5.96 & 5.7 & \\
\hline$\Delta H_{\text {hyd }}{ }_{(116)}$ & & $\begin{array}{c}-78.5 \pm 3 \\
(-53.4 \pm 8)\end{array}$ & & & \\
\hline$\Delta H_{\text {hyd }_{(211)}}$ & $-101.3 \pm 4$ & $\begin{array}{l}-80.8 \pm 5 \\
(-62.1 \pm 10)\end{array}$ & $-79.6 \pm 4$ & & $-82.5 \pm 4$ \\
\hline$\Delta H_{\text {hyd }_{(507)}}$ & & $\begin{array}{c}-81.5 \pm 4 \\
(-79.8 \pm 10)\end{array}$ & $-84.7 \pm 6$ & & \\
\hline
\end{tabular}

${ }^{\mathrm{a}}$ Reference 47.

${ }^{\mathrm{b}}$ Reference 2 and 105. 

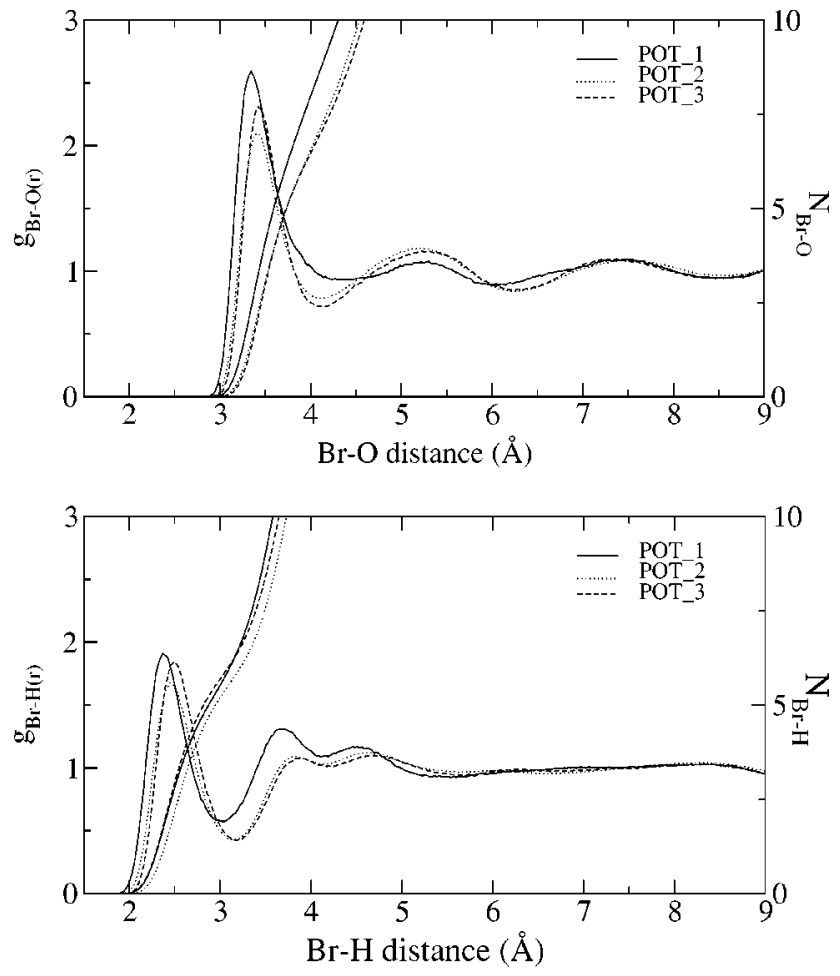

FIG. 5. $\mathrm{Br}-\mathrm{O}$ and $\mathrm{Br}-\mathrm{H}$ radial distribution functions and their running numbers for Monte Carlo simulations of $1 \mathrm{Br}^{-}$and $211 \mathrm{H}_{2} \mathrm{O}$.

similarity between $a b$ initio data at different levels $s^{42,43,68}$ shows the convergence between the level of calculation these authors employed and ours. At this level, it is shown the lack of importance in carrying out the comparison using geometries that have not been quantum-mechanical minimized at the same level of that used for the prospecting of the bromide-water system. The values in parentheses correspond to the interaction energies found when the quantummechanical geometries are allowed to relax within the force field of the interaction potentials here used. The analysis of the final structures showed that clusters minimized with POT_1, POT_2 and POT_3 evolve to arrangements where the anion is placed on the surface of the clusters formed by the water molecules (surface clusters). It is worth pointing out that despite the prospecting collected in Table III was not exhaustive, it is largely illustrative of the capability of the potentials to reproduce $a b$ initio calculations.

In order to get a better understanding of the topology of the bromide ion-water potential energy surfaces, we have explored wider regions for $n=1-6$ with POT_i $(\equiv 1,2,3)$. With this procedure we are not interested in finding the absolute minima of the $\left[\mathrm{Br}\left(\mathrm{H}_{2} \mathrm{O}\right)_{n}\right]^{-}$cluster, but rather we want to know if the minima obtained present substantial differences attending to the interaction potential used. The minimization process was performed taking different structures for each $n$ value. Figure 3 collects some of the most representative minima obtained. The comparison of the results shows that the three potentials favor the surface structures versus the internal ones. POT_1 presents the singularity of being more attractive in all cases.

Monte Carlo simulations of $\left[\mathrm{Br}\left(\mathrm{H}_{2} \mathrm{O}\right)\right]^{-}{ }_{n} \quad(n$ $=1-6,10,15,20)$ clusters at $298 \mathrm{~K}$ were carried out in order to compare with the experimental $\Delta H_{\text {bind }}$ data. ${ }^{53,62}$ Although there are no experimental estimations for $n>6$, we have performed an extrapolation assuming the additivity shown by the known values, i.e., $n \leqslant 6$. Average $\Delta H_{\text {bind }}$ has been plotted versus the number of water molecules in Fig. 4. This comparison implies the inclusion of the enthalpy correction to the theoretical values. To this end, we have taken the approximation of ideal gas behavior for the formation reaction of the ionic cluster, then $\Delta(\mathrm{PV}) \sim \Delta \mathrm{nRT}$. There is a good correlation between the three potentials and the experimental data (discrepancies below 20\%). The agreement with the experimental results shows the capability of the potentials to reproduce empirical information not used in their development. POT_1 results are closer to the experimental values than POT_2 and POT_3 ones. It should be considered that classical simulations cannot be effectively parametrized to describe quantum behavior, such as tunneling effects and zero-point energy effects, resulting in a lower estimate of $\Delta H .^{100}$ The energy gap observed in Fig. 4 is therefore an indication of the methodological uncertainty implicit to the procedure here adopted. To make a direct comparison with experiments, a quantum treatment of nuclear motion, especially of the hydrogen atoms, would be required in the simulation. That goal can be obtained, for instance, by means of a path-integral scheme ${ }^{101-104}$ but this goes beyond the aim of the present study, that tries to supply general procedure to allow the simulations of systems with a number of particles large enough to mimic aqueous solutions.

\section{B. Numerical simulations of $\mathrm{Br}^{-}$hydration}

The Monte Carlo results considering 1 bromide anion and 211 water molecules under the simulation conditions previously described were taken from a statistical sampling of $3.5 \mathrm{G}$ configurations. Runs of $2.5 \mathrm{G}$ configurations for a sample of pure water were also carried out. Table IV collects the most relevant structural and energetic results after analyzing the Monte Carlo simulations. In the next two sections, they are discussed in detail.

\section{Structural results}

Regarding the structural properties of the ionic solution, the $\mathrm{Br}-\mathrm{O}$ and $\mathrm{Br}-\mathrm{H}$ radial distribution functions are shown in Fig. 5, as well as the running integration numbers around the bromide ion. The three potentials developed are able to reproduce the experimentally determined $\mathrm{Br}-\mathrm{O}$ distance $^{105,106}$ that lies between 3.30 and $3.43 \AA$. POT_1 overestimates the number of water molecules in the first solvation shell $(\sim 10)$, whereas POT_2 $(\sim 7.2)$ and POT_3 $(\sim 7.0)$, yield a better agreement with the experimentally determined hydration number, i.e., between six and eight water molecules. ${ }^{105}$ The $\mathrm{Br}-\mathrm{H}$ RDF shows a first peak centered at 2.4-2.55 $\AA$ that extends up to 3.0-3.15 $\AA$ and integrates $\sim$ six hydrogen atoms. This indicates that the water molecules forming the first hydration shell orient, as expected, only one hydrogen atom towards the bromide. The second peak centered at ca. $3.5 \AA$ extends to $4.2 \AA$ and integrates to ca. 15 atoms; that is, in this region there are hydrogen atoms from the first hydration shell water molecules, coexisting with hydrogen atoms from the second hydration shell. 
a)

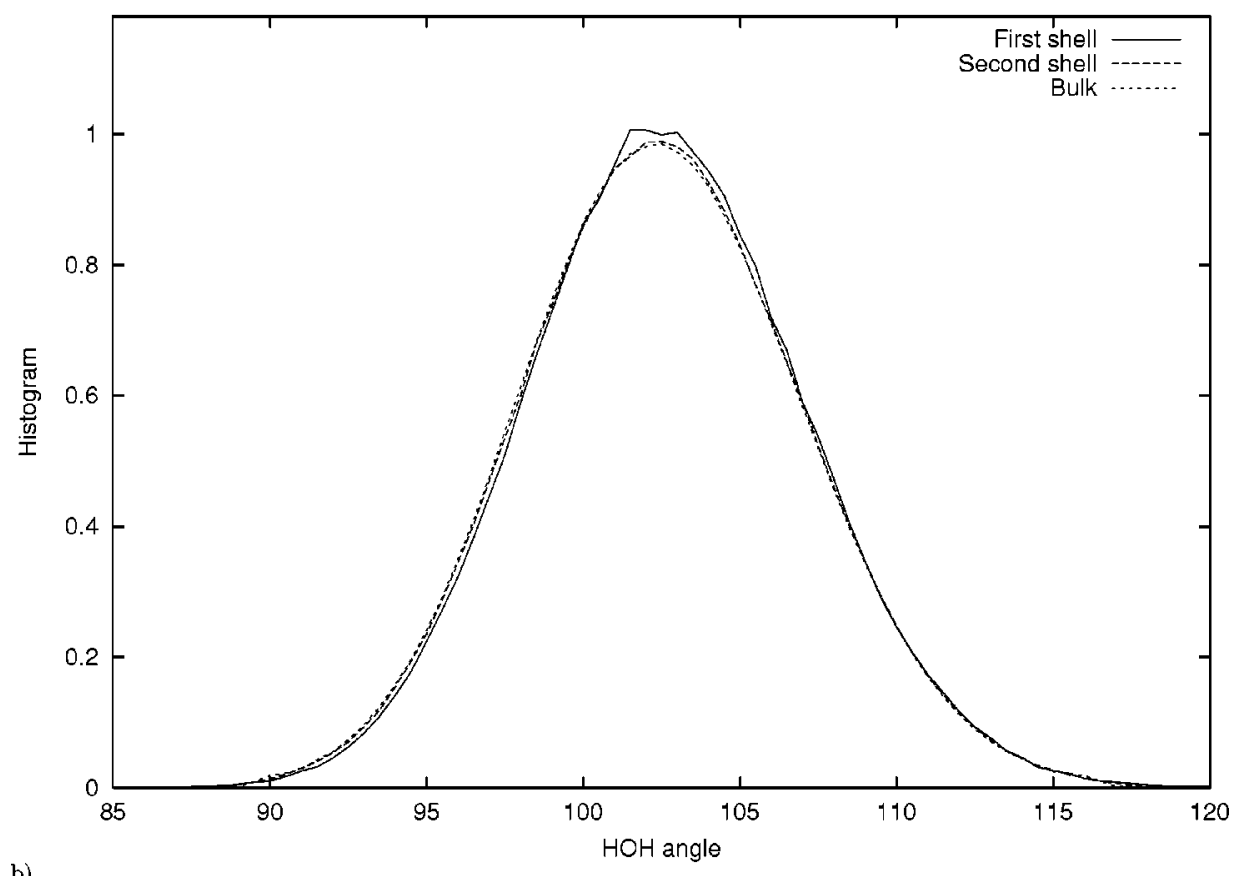

b)

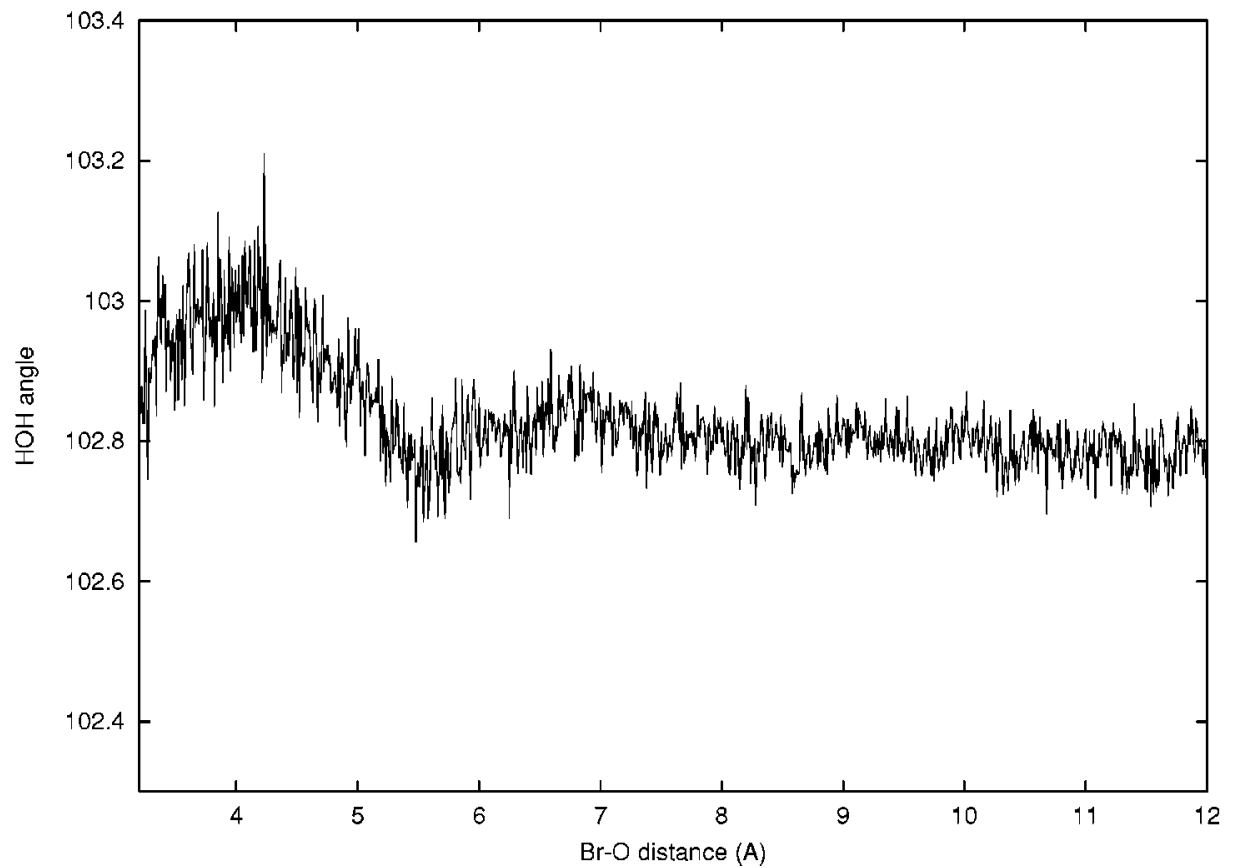

FIG. 6. HOH angle of water molecules obtained from $\mathrm{MC}$ simulations $\left(1 \mathrm{Br}^{-}\right.$and $\left.506 \mathrm{H}_{2} \mathrm{O}\right)$ with POT_2. Top: Histogram of the angle by shells, normalized to the respective maximum frequencies. Bottom: Average $\mathrm{HOH}$ angle as a function of the $\mathrm{Br}-\mathrm{O}$ distance.
Simulations were performed taking into account a relatively small system ( 1 bromide anion +211 water molecules). In order to investigate the size-consistency of the results, a larger system containing 507 water molecules was tested. The box dimensions were adjusted to keep the same density as in the previous simulations. The radial distribution functions were very similar to those previously obtained from the smaller system, thus showing that the closest hydration structure around the bromide anion was already welldefined for the small systems.

The effect of the anion on the intramolecular geometry of the water molecules and the framework of the hydrogen bonds in its vicinity, have also been investigated. Statistically independent samples of $60 \mathrm{M}$ configurations were analyzed, using POT_2 for a system containing 1 bromide anion +507 water molecules. Along the different trajectories the following parameters were studied:

(a) The water molecule angle. Figure 6(a) shows the histogram of the geometrical parameter in different shells of the solution, and Fig. 6(b) shows the dependence of the angle with the $\mathrm{Br}-\mathrm{O}$ distance. The dispersion of the values in the first and second solvation shells is similar to that of pure water, thus showing that the distortion produced by the anion on water molecule is negligible [Fig. 6(b) illustrates that the largest difference of water angle with respect to the bulk value is not more than $0.3^{\circ}$ in the first hydration shell]. These results differ from those obtained by Baik et al. ${ }^{30}$ for the fluoride anion, where the halide-water interactions are stron- 

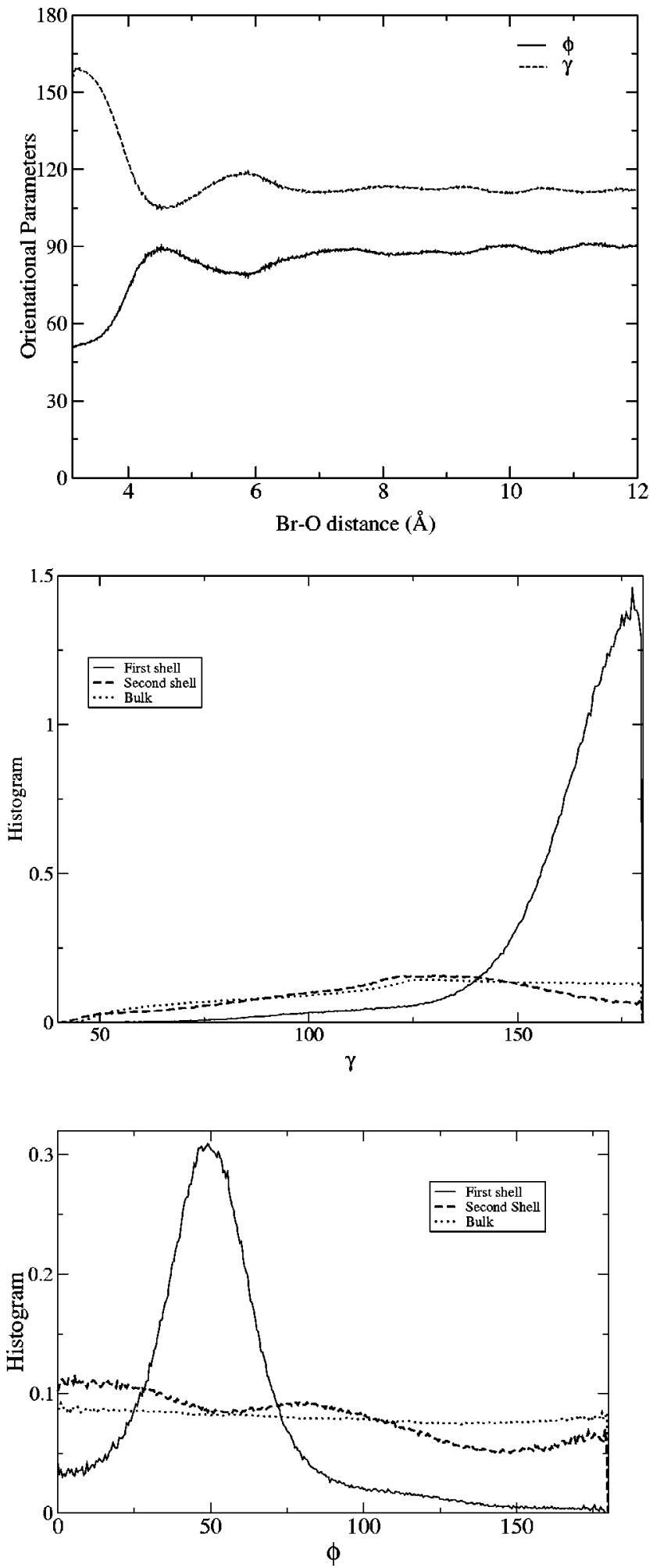

FIG. 7. Orientational parameters of water molecules with respect to the bromide anion, as defined in Scheme 1: $\gamma$ is the $\mathrm{BrOH}$ angle, and $\phi$ is the angle between the dipole moment of a water molecule and the vector connecting the anion and the oxygen atom. Average angles $\gamma$ and $\phi$ obtained with POT_2 as functions of the $\mathrm{Br}-\mathrm{O}$ distance (top graph). Histograms of the angle $\bar{\gamma}$ (middle graph) and of the angle $\phi$ (bottom graph) by hydration shells.

ger than in the case of the bromide anion, and systematic changes of the $\mathrm{HOH}$ angle with the distance to the anion were observed. (b) Orientational parameters: defining by means of the tilt angle, $\phi$, formed by the water dipole moment and the $\mathrm{Br}-\mathrm{O}$ vector, and the $\angle \mathrm{BrHO}$ angle, $\gamma$ (Scheme 1$)$. A value of $\gamma$ close to $180^{\circ}$ indicates quasi-linear hydrogen bonding. As we are dealing with anion hydration, the information that can be extracted from the tilt angle analysis is less clear than when cation hydration is considered. In the latter, a value of $\phi$ close to $0^{\circ}$ implies that the ion and the water molecule are contained in the same plane, otherwise the ion is out of the water molecular plane. However, in the case of the anions the tilt angle gives information about both the outward of the ion from the molecular plane and the orientation of the water molecule respect to the bromide anion in the molecular plane. A value of $\phi \sim 90^{\circ}$ is the asymptotic value for an ion-independent structure. The orientational features of water structure when it moves further off from the bromide anion are represented at the top of Fig. 7. One can clearly distinguish the first and second solvation shells from the bulk. Between 3.2 and $3.5 \AA$, the $\phi$ angle has a value close to $50^{\circ}$ indicating a clear orientation of water molecules with respect to the bromide anion. This result agrees with those obtained by Raugei and Klein ${ }^{47}$ and Tuñon et al. ${ }^{61}$ in recent theoretical studies about the bromide solvation. This fact is also supported by the values of the angle $\gamma \sim 160^{\circ}$. At the middle and bottom of Fig. 7 the histograms of $\gamma$ and $\phi$ angles when a shell-like analysis is performed are plotted. The analysis of these plots reveals that most of water molecules in the first hydration shell present an orientation that is given by $\phi$ $\sim 50^{\circ}$ and $\gamma \sim 175^{\circ}$. Contrary, the value of these angles for the second solvation shell and the bulk are much spreader and maxima are displayed toward the values corresponding to non-directed orientation with respect to the anion.

(c) Dependence of the water dipole moment with the $\mathrm{Br}-\mathrm{O}$ distance. This information, allowed by the use of a polarizable water model, is shown in Fig. 8. Only the firstshell water molecules present dipole moment values differing from that of the pure water. At long distances, the water dipole moment of the pure water is well reproduced. On the contrary, Klein and Raugei ${ }^{47}$ do not find differences in the average value of this property attending to the solvation shell considered. However, the reduced number of water molecules (31) used in their AIMD simulations could be the origin of the discrepancy. In fact, they underline the interest in examining the evolution of the properties with the increase of the system size.

The bromide dipole moment can also be computed because of the polarizable character of the anion. For the case of POT_2 a value in the interval [0.8-0.9] D is obtained. This value agrees with recent AIMD results ${ }^{47}$ which suggest a relation between the induced net dipole moment with an asymmetry of the first solvation shell. Tuñon et al. ${ }^{61}$ in a QM/MM study of bromide aqueous solutions obtain a much smaller dipole moment $(0.21 \mathrm{D})$. Raugei and Klein ${ }^{47}$ explain the discrepancy with $\mathrm{QM} / \mathrm{MM}$ results on the basis of the nonpolarizable water model used by Tuñon et al. ${ }^{61}$. This may responsible for a more symmetric hydration structure around the anion. Bearing this idea in mind, the estimated value of the bromide dipole moment can be used as a measurement of the symmetry of the first solvation shell. 

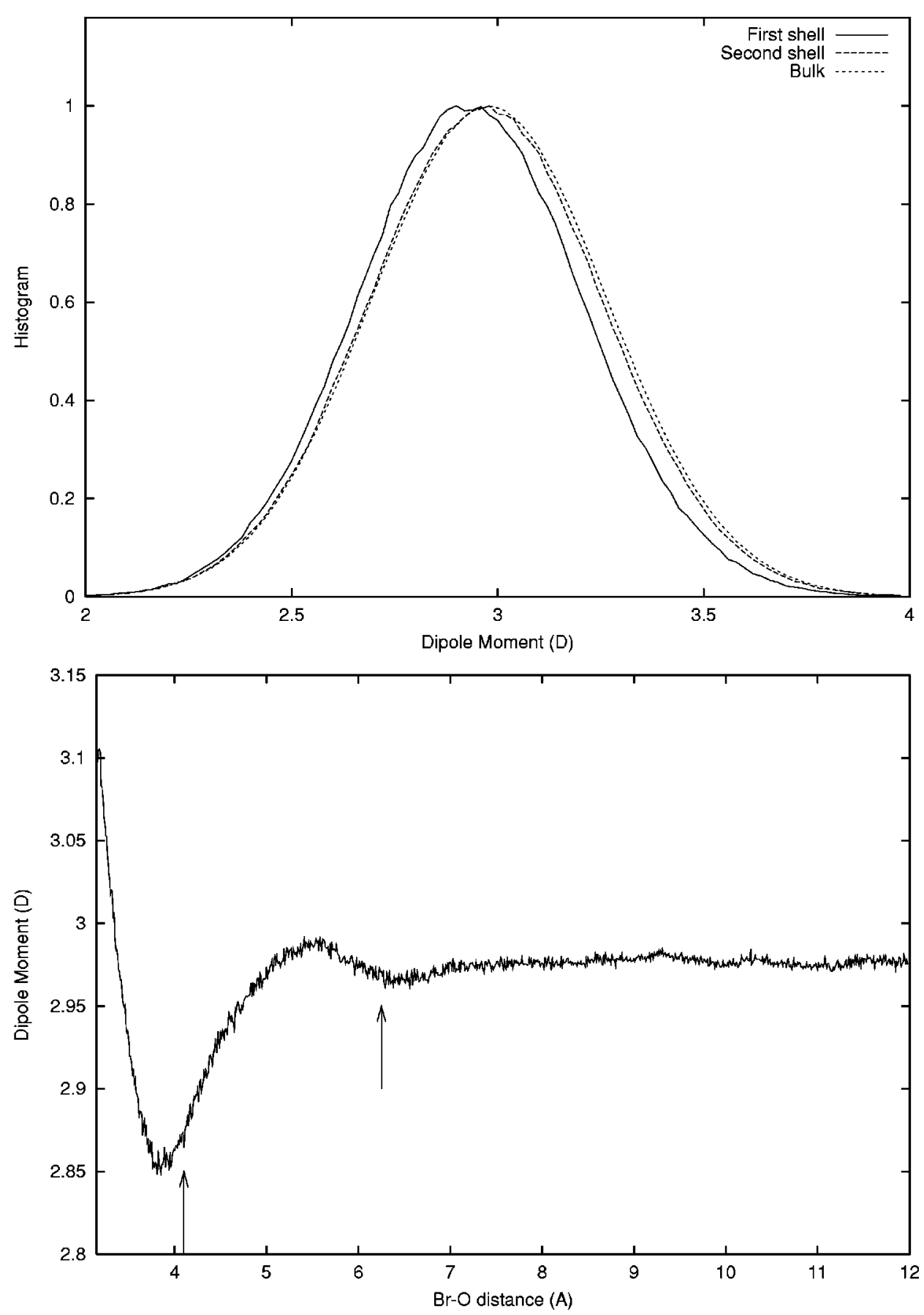

FIG. 8. Top: Histogram of the water dipole moment by hydration shells, normalized to their respective maximum frequencies. Bottom: Average per-molecule dipole moment of water as a function of the $\mathrm{Br}-\mathrm{O}$ distance. The arrows indicate the different solvation shells.

Fig. 8

\section{Energetic results}

The bromide-water potentials and system sizedependence on hydration enthalpy of the bromide anion, $\Delta H_{\text {hyd. }}\left(\mathrm{Br}^{-}\right)$, have been studied (Table IV). The experimental extrapolation to infinite dilution ${ }^{2}$ is $-82.5 \pm 4 \mathrm{kcal} / \mathrm{mol}$. Similar to the structural results, POT_1 is not able to reproduce experimental hydration energy estimation, overestimating the magnitude, but the rest of potentials are able to reproduce the experimental value within a $5 \%$ of uncertainty.

In order to know the capability of Ewald summation treatment to provide size consistent hydration enthalpy for ionic systems, we have performed three Monte Carlo simulations considering different number of particles: (A) $\mathrm{Br}^{-}$
$+116 \mathrm{H}_{2} \mathrm{O}$, (B) $\mathrm{Br}^{-}+211 \mathrm{H}_{2} \mathrm{O}$ and (C) $\mathrm{Br}^{-}+507 \mathrm{H}_{2} \mathrm{O}$. Production runs were obtained with $250 \mathrm{M}$ configurations. To estimate the hydration enthalpy of the bromide anion, the reference energy of liquid water was obtained for the corresponding simulation of a MCDHO water system with the appropriate number of molecules. All the simulations were performed using the POT_2 potential under the same conditions described above. $\Delta H_{\text {hyd }}$ values were found to be the same, bearing in mind the standard deviations of the simulations $(3-5 \mathrm{kcal} / \mathrm{mol})$. These values must be compared with the estimated $\Delta H_{\text {hyd }}$ of simulations without the Ewald summation treatment for the long-range interactions. In these cases, a clear size-dependency is found, $\Delta H_{\text {hyd }}$ changing 
from -53.4 to $-79.8 \mathrm{kcal} / \mathrm{mol}$. Thus, we can conclude that the Ewald sum with the correction term ${ }^{92}$ to account for the non-neutral character of the system produces results that are consistent with the number of water molecules considered.

From the thermodynamic and structural results derived from the simulations for a large number of particles, we concluded that POT_1 is diverging from experimental values, becoming too much attractive and overestimating the coordination number of water molecules around bromide anion. The inability of POT_1 to reproduce the experimental data shows the importance of including the BSSE corrections. Results from POT_2 and POT_3 are in good agreement with experimental data. This concordance is also supported by the results obtained from minimizations. The resemblance between POT_2 and POT_3 corroborates the tendency observed in the three-body contributions, viz. the inclusion of the electron correlation in the nonadditivity contributions does not affect the thermodynamic and energetic results obtained. In spite of the fact that four-body contributions were not included in the development of POT_2, their absence does not have an influence either on the clusters or the hydration studies; this fact is not surprising since despite not being fitted, it is able to yield a good reproduction of fourbody terms a posteriori.

\section{CONCLUDING REMARKS}

In summary, we have developed a set of first-principles bromide ion-water interaction potentials on the basis of polarizable model for water molecules and the halide. Combination of them with a MCDHO water-water potential allows minimizations of $\left[\mathrm{Br}\left(\mathrm{H}_{2} \mathrm{O}\right)_{n}\right]^{-}$clusters in gas phase, as well as simulations of these clusters at $298 \mathrm{~K}$ and bromide aqueous solutions. The comparison with experimental data defines a minimum number of ingredients needed for a reliable potential: BSSE correction of two-body interactions, inclusion of three-body contributions and a polarizable character of the particles. These elements warranty an accurate description of the physico-chemical properties of the system both in gas phase and aqueous solution. Our energetic and structural results show how interaction potentials based on first-principles calculations are able to reproduce properties of ionic clusters derived from gas phase studies as well as properties of ionic aqueous solutions. Contrary to what some authors have reported, ${ }^{11} a b$ initio methods are accurate enough to build models for both clusters and condensed phase calculations. No particular parametrization has been included to account for the change of state from gas phase to solutions.

Anion presence does not affect the intramolecular geometry of water molecules. This supports the use of a rigid model of water molecules as an approach to describe the bromide hydration. It has been recently shown ${ }^{107}$ that a rigid version of the MCDHO potential having the average geometry of the molecule in the liquid, yields similar results to the flexible model. Dipole moment of the water molecules is perturbed by ca. $5 \%$.

No size dependence for the hydration enthalpy when the Ewald sum treatment is properly applied was found. Com- puted $\Delta H_{\text {hyd }}$ is in the interval $[-78.5,-81.5] \mathrm{kcal} / \mathrm{mol}$ when going from 121 to 512 water molecules, which agrees well with the experimental estimation ${ }^{2}$ of -82.5 $\pm 4 \mathrm{kcal} / \mathrm{mol}$. In all cases, but POT_1, the $\mathrm{Br}-\mathrm{O}$ coordination number is between 6 and 8 and the $\mathrm{Br}-\mathrm{O}$ distance lies within the experimentally determined range, [3.30-3.43] A.

The development of a MCDHO-type bromide anionwater interaction potential implies the consideration of the instantaneous polarizability for the bromide anion. Alternative developments in which the ion polarizability was considered in an average way would get insight into the nature of the main factors dominating the halide-water interactions in both gas phase clusters and aqueous solutions. Studies in that direction are in progress.

\section{ACKNOWLEDGMENTS}

Spanish DGICYT (PB98-1153) and Junta de Andalucía (group FQM 282) are thanked for financial support, as well as DGAPA-UNAM IN110399 and CONACYT G33362-E. The CICA (Centro Informático Científico de Andalucía) is thanked for providing part of the computational time of this work. R.A.E. thanks I.O.B. and H.S.M. for their warm hospitality during her stay at Cuernavaca. H.S.M. thanks E.S.M. and R.A.E. for their hospitality during his stay at Sevilla.

${ }^{1}$ B. E. Conway, Ionic Hydration in Chemistry and Biophysics. Studies in Physical and Theoretical Chemistry (Elsevier, Amsterdam, 1981), Vol. 12.

${ }^{2}$ Y. Marcus, Ion Solvation (Wiley, Chichester, 1986).

${ }^{3}$ M. P. Allen and D. J. Tildesley, Computer Simulation of Liquids (Oxford University Press, Oxford, 1987).

${ }^{4}$ D. W. Hermann, Computer Simulation Methods (Springer-Verlag, Berlin, 1990).

${ }^{5}$ E. Clementi, in Modern Techniques in Computational Chemistry (Escom, Leiden, 1990), Chap. I.

${ }^{6}$ B. Y. Simkin and I. I. Sheikhet, Quantum Chemical and Statistical Theory of Solutions: A Computational Approach (Ellis Horwood, London, 1995).

${ }^{7}$ A. Warshel, Computer Modeling of Chemical Reactions in Enzymes and Solutions (Wiley, New York, 1991).

${ }^{8}$ K. Heizinger and G. Palinkas, in The Chemical Physics of Solvation, edited by E. Dogonadze, A. Kornyshevx, and J. Ulstrup (Elsevier, New York, 1985), Chap. 4.

${ }^{9}$ P. B. Balbuena and J. M. Seminario, Molecular Dynamics: From Classical to Quantum Methods (Elsevier, Amsterdam, 1999), Vol. 7.

${ }^{10}$ A. J. Stone, The Theory of Intermolecular Forces (Clarendon, Oxford, 1996).

${ }^{11}$ P. J. van Maaren and D. van der Spoel, J. Phys. Chem. B 105, 2618 (2001).

${ }^{12}$ G. Markovich, S. Pollack, R. Giniger, and O. Chesnovsky, Z. Phys. D Atom. Mol. 26, 98 (1993).

${ }^{13}$ G. Markovich, S. Pollac, R. Giniger, and O. Cheshnovsky, J. Chem. Phys. 101, 9344 (1994).

${ }^{14}$ L. Perera and M. L. Berkowitz, J. Chem. Phys. 95, 1954 (1991).

${ }^{15}$ L. Perera and M. L. Berkowitz, J. Chem. Phys. 96, 8288 (1992).

${ }^{16}$ L. Perera and M. L. Berkowitz, J. Chem. Phys. 99, 4222 (1993).

${ }^{17}$ L. Perera and M. L. Berkowitz, J. Chem. Phys. 100, 3085 (1994).

${ }^{18}$ L. S. Sremaniak, L. Perera, and M. L. Berkowitz, J. Phys. Chem. 100, 1350 (1996).

${ }^{19}$ L. S. Sremaniak, L. Perera, and M. L. Berkowitz, Chem. Phys. Lett. 218, 377 (1994).

${ }^{20}$ J. E. Combariza, N. R. Kestner, and J. Jortner, Chem. Phys. Lett. 203, 423 (1993)

${ }^{21}$ J. E. Combariza and N. R. Kestner, J. Phys. Chem. 98, 3513 (1994).

22 J. E. Combariza, N. R. Kestner, and J. Jortner, J. Chem. Phys. 100, 2851 (1994). 
${ }^{23}$ J. E. Combariza, N. R. Kestner, and J. Jortner, Chem. Phys. Lett. 221, 156 (1994).

${ }^{24}$ J. E. Combariza and N. R. Kestner, J. Phys. Chem. 99, 2717 (1995).

${ }^{25}$ L. X. Dang and B. C. Garret, J. Chem. Phys. 99, 2972 (1993).

${ }^{26}$ L. X. Dang, J. Chem. Phys. 110, 1526 (1999).

${ }^{27}$ S. S. Xantheas, J. Am. Chem. Soc. 117, 10373 (1995).

${ }^{28}$ S. S. Xantheas, J. Phys. Chem. 100, 9703 (1996).

${ }^{29}$ O. M. Cabarcos, C. J. Weinheimer, J. M. Lisy, and S. S. Xantheas, J. Chem. Phys. 110, 5 (1999).

${ }^{30}$ K. Baik, J. Kim, D. Majumadar, and K. M. Kim, J. Chem. Phys. 110, 9116 (1999).

${ }^{31}$ S. J. Vaughn, E. V. Akhmastkaya, M. A. Vincent, A. J. Masters, and I. H. Hillier, J. Chem. Phys. 110, 4338 (1999).

${ }^{32}$ R. A. Bryce, M. A. Vincent, N. O. J. Malcolm, and I. H. Hillier, J. Chem. Phys. 109, 3077 (1998).

${ }^{33}$ P. Ayotte, C. G. Bailey, G. H. Weddle, and M. A. Johnson, J. Phys. Chem. A 102, 3067 (1998).

${ }^{34}$ P. Ayotte, G. H. Weddle, and M. A. Johnson, J. Chem. Phys. 110, 7129 (1999).

${ }^{35}$ P. Ayotte, G. H. Weddle, J. Kim, J. A. Kelley, and M. A. Johnson, J. Phys. Chem. A 103, 443 (1999).

${ }^{36}$ S. Lee, J. Kim, J. Park, and K. S. Kim, J. Phys. Chem. 100, 14329 (1996).

${ }^{37}$ J.-H. Choi, Y.-B. C. Keith, T. Kuwata, and M. Okumura, J. Phys. Chem. A 102, 503 (1998).

${ }^{38}$ L. X. Dang and T. M. Chang, J. Chem. Phys. 106, 8149 (1997).

${ }^{39}$ L. X. Dang and D. E. Smith, J. Chem. Phys. 99, 6950 (1993).

${ }^{40}$ I. A. Topol, G. J. Tawa, S. K. Burt, and A. A. Rashin, J. Chem. Phys. 111, 10998 (1999).

${ }^{41}$ D. Majumdar, J. Kim, and K. S. Kim, J. Chem. Phys. 112, 101 (2000).

${ }^{42}$ J. Kim, H. M. Lee, S. S. Suh, and K. S. Kim, J. Chem. Phys. 113, 5259 (2000)

${ }^{43}$ H. M. Lee, D. Kim, and K. S. Kim, J. Chem. Phys. 116, 5509 (2002).

${ }^{44}$ M. Kropman and H. J. Barker, Science 2921, 2119 (2001).

${ }^{45}$ M. L. Klein, Science 2921, 2106 (2001).

${ }^{46}$ S. Raugei and M. L. Klein, J. Am. Chem. Soc. 123, 9484 (2001).

${ }^{47}$ S. Raugei and M. L. Klein, J. Chem. Phys. 116, 196 (2002).

${ }^{48}$ S. J. Stuart and B. J. Berne, J. Phys. Chem. 100, 11934 (1996).

${ }^{49}$ S. J. Stuart and B. J. Berne, J. Phys. Chem. A 103, 10300 (1999).

${ }^{50}$ W. L. Jorgensen and D. L. Severance, J. Chem. Phys. 99, 4233 (1993).

${ }^{51}$ S. K. Searles and P. Kebarle, Can. J. Chem. 47, 2619 (1969).

${ }^{52}$ R. G. Keesee and J. A. W. Castleman, Chem. Phys. Lett. 74, 139 (1980).

${ }^{53}$ K. Hiraoka, S. Mizuse, and S. Yamabe, J. Phys. Chem. 92, 3943 (1988).

${ }^{54}$ F. K. Tanabe, N. H. Morgon, and J. M. Riveros, J. Phys. Chem. 100, 2862 (1996).

${ }^{55}$ H. Tanida, H. Sakane, and I. Watanabe, J. Chem. Soc. Dalton Trans. , 2321 (1994)

${ }^{56}$ Y. Sawa, T. Miyanaga, H. Tanida, and I. Watanabe, J. Chem. Soc., Faraday Trans. 91, 4389 (1995).

${ }^{57}$ P. D. 'Angelo, A. D. Nola, M. Mangoni, and N. V. Pavel, J. Chem. Phys. 104, 1779 (1996)

${ }^{58}$ S. L. Wallen, B. J. Palmer, D. M. Pfund, L. Fulton, M. Newville, Y. Ma, and E. A. Stern, J. Phys. Chem. A 101, 9632 (1997).

${ }^{59}$ O. M. Cabarcos, C. J. Weinheimer, T. J. Martinez, and M. Lisy, J. Chem. Phys. 110, 9516 (1999).

${ }^{60}$ C. Brooks III, J. Phys. Chem. 90, 6680 (1986).

${ }^{61}$ I. Tunón, M. T. C. Martins-Costa, C. Millot, and M. F. Ruíz-López, Chem. Phys. Lett. 241, 450 (1995).

${ }^{62}$ K. Heinzinger and G. Palinkas, Z. Naturforsch. A 32, 1137 (1977).

${ }^{63}$ K. Heinzinger, Phys. B and C 131, 196 (1985).

${ }^{64}$ S. Koneshan, J. Rasaiah, M. Lynden-Bell, and H. Lee, J. Phys. Chem. B 102, 4193 (1998).

${ }^{65}$ T. P. Lybrand, I. Ghosh, and J. A. McCammon, J. Am. Chem. Soc. 107, 7793 (1985)

${ }^{66}$ T. P. Straastma and H. J. C. Berendsen, J. Chem. Phys. 89, 5876 (1988).

${ }^{67}$ M. F. van Gunsteren and H. J. C. Berendsen, in GROMOS Manual, edited by Biomos (Library Manual, Groninger, 1987).
${ }^{68}$ R. Ayala, J. M. Martínez, R. R. Pappalardo, and E. Sánchez Marcos, J. Phys. Chem. A 104, 2799 (2000).

${ }^{69}$ H. Saint-Martin, J. Hernandez-Cobos, M. I. Bernal-Uruchurtu, I. OrtegaBlake, and H. J. C. Berendsen, J. Chem. Phys. 113, 10899 (2000).

${ }^{70}$ S. A. Clough, Y. Beers, G. P. Klein, and L. S. Rothman, J. Chem. Phys. 59, 2254 (1973).

${ }^{71}$ J. Verhoeven and A. Dymanus, J. Chem. Phys. 52, 3222 (1970).

${ }^{72}$ W. F. Murphy, J. Chem. Phys. 67, 5877 (1977).

${ }^{73}$ S. J. Vaughn, E. V. Akhmastkaya, M. A. Vincent, A. J. Masters, and I. H. Hillier, J. Chem. Phys. 110, 4338 (1999).

${ }^{74}$ X. Periole, D. Allouche, J.-P. Daudey, and Y.-H. Sanejouand, J. Phys. Chem. B 101, 5018 (1997).

${ }^{75}$ X. Periole, D. Allouch, A. Ramírez-Solís, I. Ortega-Blake, J.-P. Daudey, and Y.-H. Sanejouand, J. Phys. Chem. B 102, 8579 (1998).

${ }^{76}$ M. J. Frisch, G. W. Trucks, H. B. Schlegel et al., GAUSSIAN 98, Revision A.3, Gaussian, Inc., Pittsburgh, PA, 1998.

${ }^{77}$ C. W. Bauschlicher Jr., H. F. Schaefer III, and P. S. Bagus, J. Am. Chem. Soc. 99, 7106 (1977)

${ }^{78}$ M. Kaupp, P. v. R. Schleyer, H. Stoll, and H. Preuss, J. Am. Chem. Soc. 113, 6012 (1991)

${ }^{79}$ T. H. Dunning, J. Chem. Phys. 90, 1007 (1989).

${ }^{80}$ R. A. Kendall, T. H. Dunning, and R. J. Harrison, J. Chem. Phys. 96, 6796 (1992).

${ }^{81}$ C. A. Deakyne, in Molecular Interactions: From van der Waals to Strongly Bound Complexes, edited by S. Scheiner (Wiley, New York, 1997).

${ }^{82}$ S. F. Boys and F. Bernardi, Mol. Phys. 19, 553 (1970).

${ }^{83}$ A. K. Kartz, J. P. Glusker, S. A. Beebe, and C. W. Bock, J. Am. Chem. Soc. 118, 5752 (1996)

${ }^{84}$ C. W. Bock, A. K. Kartz, and J. P. Glusker, J. Am. Chem. Soc. 117, 3754 (1995).

${ }^{85}$ K. Szalewicz, S. J. Cole, W. Kolos, and R. J. Barlett, J. Chem. Phys. 89, 3662 (1988)

${ }^{86}$ M. Pavlov, P. E. M. Siegbahn, and M. Sandstrom, J. Phys. Chem. A 102, 219 (1998)

${ }^{87}$ V. F. Lotrich and K. Szalewicz, J. Chem. Phys. 106, 9668 (1997).

${ }^{88}$ L. X. Dang, J. E. Rice, J. Caldwell, and P. A. Kollman, J. Am. Chem. Soc. 113, 2481 (1991).

${ }^{89}$ L. X. Dang, J. Chem. Phys. 96, 6970 (1992).

${ }^{90}$ MCHANG program, This library is available upon request at jorge@ fis.unam.mx

${ }^{91}$ J. Kim and K. S. Kim, J. Chem. Phys. 109, 5886 (1998).

${ }^{92}$ J. E. Roberts and J. Schnitker, J. Phys. Chem. 99, 1322 (1995).

${ }^{93}$ M. Leslie and M. J. Gillan, J. Phys.: Condens. Matter 18, 973 (1985).

${ }^{94}$ J. D. Madura and B. M. Pettit, Chem. Phys. Lett. 150, 105 (1988).

${ }^{95}$ L. Perera, U. Essmann, and M. L. Berkowitz, J. Chem. Phys. 102, 450 (1995).

${ }^{96}$ G. Hummer, L. R. Pratt, and A. E. Garcia, J. Phys. Chem. 100, 1206 (1996).

${ }^{97}$ G. Hummer, L. R. Pratt, A. E. Garcia, and G. Shekhar, J. Phys. Chem. B 102, 3841 (1998)

${ }^{98}$ J. Aqvist and T. Hansson, J. Phys. Chem. B 102, 3837 (1998).

${ }^{99}$ N. Metropolis, A. W. Rosenbluth, R. M. Rosenbluthnd, A. H. Teller, and E. Teller, J. Chem. Phys. 21, 1087 (1953).

${ }^{100}$ H. Gai, L. X. Dang, G. K. Schenter, and B. C. Garret, J. Phys. Chem. 99, 13303 (1995)

${ }^{101}$ R. P. Feynman and A. R. Hibbs, Quantum Mechanics and Path Integrals (McGraw-Hill, New York, 1965).

${ }^{102}$ M. Parrinello and A. Rahman, J. Chem. Phys. 80, 860 (1984).

${ }^{103}$ R. A. Kuharski and P. J. A. Rossky, Chem. Phys. Lett. 103, 357 (1984).

${ }^{104}$ H. A. Stern and B. J. Berne, J. Chem. Phys. 115, 7622 (2001).

${ }^{105}$ H. Ohtaki and T. Radnai, Chem. Rev. 93, 1157 (1993).

${ }^{106}$ P. A. Bergström, J. Lindren, and O. Kristiansson, J. Phys. Chem. 95, 8575 (1995).

${ }^{107}$ B. Hess, H. Saint-Martin, and H. C. J. Berendsen, J. Chem. Phys. 116, $9602(2002)$. 